Bio - grafía. Escritos sobre la Biología y su Enseñanza. ISSN 2027-1034

Edición Extraordinaria. p.p. 909 - 917

Memorias del IX Encuentro Nacional de Experiencias en Enseñanza de la Biología y la Educación Ambiental. IV Congreso Nacional de Investigación en Enseñanza de la Biología.

\title{
LA BIOLOGÍA EN LA SECUNDARIA DE LA ESCUELA SOCIALISTA EN MÉXICO (1934 $-1940)$
}

\section{Vicente Paz Ruiz ${ }^{1}$ María de la Luz Martínez Hernández ${ }^{2}$}

\section{RESUMEN}

El objetivo de este texto es argumentar que el currículo para la enseñanza de las Ciencias Biológicas en la escuela secundaria socialista (1934 - 1940), sienta las bases del diseño de los subsecuentes programas de dicha asignatura en México. Se siguió una metodología de investigación documental, empleando los informes anuales de la Secretaría de Educación Pública de los años treinta, se organizó la información partiendo de la filosofía, el nivel de estudio, el área y los contenidos en; escuela socialista, escuela secundaria, ciencias naturales, ciencias biológicas. Se analizó la estructura de los cursos de Ciencias Biológicas para secundaria, retomando dos de las categorías anteriores; lo filosófico y contenidos y añadiendo lo pedagógico. En lo filosófico era materialista, en lo pedagógico trabaja por áreas que llama complejos y tiene influencias del pragmatismo de Dewey y la escuela práctica-socialista de Makarenko, en los contenidos se dividía en el estudio de la naturaleza y en el estudio del hombre. De los cuatro paradigmas de la Biología (Ledesma, 1999) se atienden dos, teoría celular y homeostasis, evolución se percibe como enfoque y se deja de lado genética.

PALABRAS CLAVE: Biología, Educación, Ciencias Biológicas, Currículum, Escuela secundaria

\section{RESUMEN}

The aim of this paper is to argue that the bases of Biological Sciences curriculum, subsequent educational programs and its design were stablished in the socialist secondary school in Mexico from 1934 to 1940 . A documentary research was conducted by using annual reports provided by the Mexican Secretariat of Public Education from the thirties. This information was organized in the socialist, secondary, biological and natural sciences schools. The Biological Science structure of the course for secondary school was analyzed, resulting in three different categories; the philosophical, educational and the content area. It was materialistic in the philosophical area, respect to the educational area, it was divided into various parts, for instance, the study of nature and the study of man. Only two theories from the four paradigms of Biology proposed by Ledesma (1999) are put on the spot; the cell theory and homeostasis. Evolution is only perceived as an approach which is left on the side of genetics.

\footnotetext{
${ }^{1}$ Universidad Pedagógica Nacional de México, Unidad 094 Centro,vpaz@upn.mx

${ }^{2}$ SEP - Coordinación sectorial, Secundarias, UPN Doctorado fluzma@hotmail.com
} 
Bio - grafía. Escritos sobre la Biología y su Enseñanza. ISSN 2027-1034

Edición Extraordinaria. p.p. 909 - 917

Memorias del IX Encuentro Nacional de Experiencias en Enseñanza de la Biología y la

Educación Ambiental. IV Congreso Nacional de Investigación en Enseñanza de la Biología.

KEY WORDS: Biology, Education, Biological Sciences, Curriculum, Secondary school.

\section{INTRODUCCIÓN}

En este escrito se habla sobre el proyecto de escuela socialista, su propuesta para la enseñanza de la ciencia en secundaria y particularmente sobre el programa de ciencias biológicas de dicho nivel. La educación socialista nació para la escuela secundaria formalmente hasta 1939, fue derogada en lo curricular en 1942 y en la constitución en 1946. Trabajos previos sobre el proyecto educativo socialista del General Lázaro Cárdenas del Río, como el de Gilly (1994) lo abordan como una utopía, Kay Vaughan (1982, 2001) aborda la filosofía y logros de la política educativa del cardenismo, de artículos sobre la importancia del cardenismo en su cruzada educativa a favor de los campesinos e indígenas, se marcan sólo algunos (Reyes, 1983; Leco, 2000; Maldonado, 2008). Montes de Oca (2007), realiza un análisis de los libros de texto del cardenismo para niños de escuelas primarias urbanas y rurales. Zorrilla (2004) hace una apretada síntesis de la historia de la escuela secundaria, pero esa generalidad le impide abordar aspectos finos del currículo socialista. Ynclán (1998), coordina un texto donde se habla de la historia, retos y prospectiva de la educación secundaria en México, pero deja de lado la enseñanza de la Biología.

En todas las obras citadas se refiere que en 1934 el analfabetismo no había mejorado mucho en el país respecto de inicios del siglo XX, $80 \%$ de la población no sabía leer ni escribir y era eminentemente rural $(82.5 \%)$, la tasa de natalidad era del 44.5 y la de mortalidad de 124 niños ambos por cada millar (INEGI, 2008), la educación sexual impulsada por Bassols que desde la escuela buscaba incidir en estas condiciones fue frenada por el clero y los conservadores, la sanidad promovida desde la oficialidad era atacada, pero en fondo se buscaba deslegitimar los avances de la ciencia y del progreso ya que el de Bassols era un intento de una transformación mental del pueblo enraizada en esquemas tradicionales dogmáticos promovidas por la iglesia (Del Castillo, 2000).

Narciso Bassols como secretario de educación redacta lo que sería el nuevo artículo $3^{\circ}$ constitucional, base ideológica de la escuela socialista, las modificaciones fueron aprobadas por la cámara de diputados el $1^{\circ}$ de diciembre de 1934 y ejecutado durante el mandato de Lázaro Cárdenas del Río. Señalaba que la educación que imparta el estado será socialista y además de excluir toda doctrina religiosa, combatirá el fanatismo y los prejuicios, para lo cual la escuela organizará sus enseñanzas y actividades en forma que permita crear en la juventud un concepto racional y exacto del universo y de la vida social (IIJ, 2009).

En 1934 la escuela secundaria se adhiere al plan socialista, buscando el ideal de adquirir un carácter coeducativo, pre vocacional, democrático, popular, socialista, racionalista y sobre todo práctico. El Programa de Educación Pública de 1935 señalaba los ideales y principios de la educación socialista, en 1936 se declara la gratuidad de la educación secundaria y se marcó el sentido práctico de la misma, señalándola como un centro de preparación técnica del alumno para el trabajo (Zorrilla, 2004). 
Bio - grafía. Escritos sobre la Biología y su Enseñanza. ISSN 2027-1034

Edición Extraordinaria. p.p. 909 - 917

Memorias del IX Encuentro Nacional de Experiencias en Enseñanza de la Biología y la

Educación Ambiental. IV Congreso Nacional de Investigación en Enseñanza de la Biología.

En condiciones precarias como las del México del primer tercio del siglo XX, la enseñanza de la ciencia, pareciera que estaba alejada de la realidad, por ello nos preguntamos ¿cuál fue el enfoque, orientación y estructura que tuvo la enseñanza de la ciencia en la educación secundaria durante el gobierno del general Lázaro Cárdenas del Río, para convertirse en uno de los principales promotores del laicismo?

De ahí que nuestro objetivo fuese conocer la propuesta curricular para la enseñanza de las ciencias biológicas en la escuela secundaria de la escuela socialista, su organización de contenidos, lógica y fundamentos tanto filosóficos como conceptuales.

\section{METODOLOGÍA}

Se siguió una metodología de investigación documental, se revisó material de la enseñanza de la Biología en la escuela secundaria, tomado de los informes anuales de la Secretaría de Educación Pública (SEP) de los años treinta, localizados en la biblioteca "Luis Guevara Ramírez", que conserva documentos históricos de lo que fue la Escuela Normal Superior de México, centro de formación de maestros de Biología para educación secundaria. Se vaciaron tres tipos de información de los documentos; textual, paráfrasis y síntesis. Se discriminó la información a partir de los temas; escuela socialista, escuela secundaria, ciencias naturales, ciencias biológicas. Posteriormente se codificaron los datos triangulando por medio de listados todo lo referido a cada tema encontrado en diversas fuentes, contrastándolas y descartando las que no eran consistentes, priorizando como criterio el currículo mismo de Ciencias Biológicas del proyecto educativo socialista. El análisis partió de reconstruir la propuesta curricular de 1935 en tablas que esquematizaron la estructura de los cursos de Ciencias Biológicas para secundaria, de ellas se derivaron tres categorías, lo filosófico, pedagógico y contenidos que permitieron interpretar y dar significado al currículo.

\section{RESULTADOS}

El proyecto socialista modificó su plan de estudios para la educación secundaria, dividiéndolo por complejos, enfoques y disciplinas. La división por complejos articulaba las diferentes disciplinas que las conformaban. Se transitó de un modelo de asignaturas, a otro de "pedagogía dialéctica", globalizando áreas y evitando la dispersión por asignaturas mutando a complejos. Se alineó a la educación básica con los requerimientos de la escuela socialista. Los tres complejos fueron, de la naturaleza, de la sociedad, del trabajo. Se aprobó el 22 de noviembre de 1939 se cursaría en tres años. El Complejo del conocimiento de la naturaleza, comprendía las asignaturas de Cosmografía, Geografía, Física, Química y Ciencias biológicas (SEP, 1935, p. 205).

Los contenidos para primer año partían del origen de la planta (la semilla), el desarrollo de la misma (tropismos), su organografía, fisiología y taxonomía, esta última en el nivel de identificación. En cada tema se veía la importancia económica del órgano o función de la planta en estudio y su aplicación práctica en la vida de la comunidad (ver tabla 1). 
Bio - grafía. Escritos sobre la Biología y su Enseñanza. ISSN 2027-1034

Edición Extraordinaria. p.p. 909 - 917

Memorias del IX Encuentro Nacional de Experiencias en Enseñanza de la Biología y la Educación Ambiental. IV Congreso Nacional de Investigación en Enseñanza de la Biología.

\section{Tabla 1:}

Contenidos sintéticos del curso de Ciencias biológicas I Botánica (SEP, 1935)

\section{Ciencias biológicas I Botánica y laboratorio, 4 hrs}

\begin{tabular}{|c|c|c|}
\hline I Germinación de semillas & VI Crecimiento y tropismo & $\begin{array}{l}\text { XI Importancia económica de } \\
\text { las hojas }\end{array}$ \\
\hline $\begin{array}{l}\text { II Las semillas de } \\
\text { importancia económica }\end{array}$ & $\begin{array}{l}\text { VII Ecología de tallos y } \\
\text { raíces }\end{array}$ & $\begin{array}{l}\text { XII Reproducción de las } \\
\text { fanerógamas }\end{array}$ \\
\hline $\begin{array}{l}\text { III Conceptos científicos de } \\
\text { la vida deducido del } \\
\text { funcionamiento de la célula }\end{array}$ & $\begin{array}{l}\text { VIII Utilización de raíces y } \\
\text { tallos }\end{array}$ & $\begin{array}{l}\text { XIII Flores y frutos } \\
\text { importancia económica }\end{array}$ \\
\hline IV Absorción radical & IX Cambios gaseosos & $\begin{array}{l}\text { XIV Propagación de los } \\
\text { vegetales superiores }\end{array}$ \\
\hline V Conducción y sostén & $\begin{array}{l}\text { X Importancia de los } \\
\text { bosques y jardines }\end{array}$ & $\begin{array}{l}\text { XV Estudios morfológicos de } \\
\text { algunas plantas típicas para } \\
\text { iniciar al alumno en la } \\
\text { clasificación vegetal }\end{array}$ \\
\hline
\end{tabular}

El segundo curso Zoología y laboratorio, se dedicaba al estudio y conocimiento de los animales. Se recomendaba que se realizará un trabajo basado en la elaboración de monografías de animales tipo, cada estudio monográfico comprendería: El medio en el que vive y su distribución geográfica, el tipo de ventilación (respiración), su importancia biológica, detalles de su morfología externa, tipo de excreción, su utilidad o nocividad para el hombre, detalles de su morfología interna, el proceso de su reproducción, cuidados de los animales en su cría o al tener contacto con ellos (higiene), forma de desplazamiento (locomoción), formas de resistencia o propagación, su industrialización (importancia económica), el tipo de nutrición (alimentación), caracteres adaptativos y su mecanismo de adaptación y características ecológicas (ver tabla 2).

\section{Tabla 2:}

Contenidos sintéticos del curso de Ciencias biológicas II Zoología (SEP, 1935).

\section{Ciencias biológicas II Zoología y laboratorio $4 \mathrm{hrs}$.}

\begin{tabular}{lll}
\hline I Ameba & VI Mariposa o chapulín & XI Rana \\
II Hidra & VII Alacrán & XII Lagartija
\end{tabular}


Bio - grafía. Escritos sobre la Biología y su Enseñanza. ISSN 2027-1034

Edición Extraordinaria. p.p. 909 - 917

Memorias del IX Encuentro Nacional de Experiencias en Enseñanza de la Biología y la

Educación Ambiental. IV Congreso Nacional de Investigación en Enseñanza de la Biología.

III Tenia, Cisticercos

IV Lombriz intestinal

V Camarón o acocil
VIII Caracol

IX Erizo o estrella de mar

X Carpa
XIII Paloma

XIV Conejo

Los dos cursos previos se refieren a la Biología pura, si bien vista desde un enfoque evolucionista, no se descuida lo práctico y económico. Para el caso del tercer curso, sin perder de vista todas aquellas consideraciones generales antes mencionadas, se dedicaba a la enseñanza de la anatomía y fisiología humanas, así como los principios elementales de la higiene, buscando proveer al alumno de aquellos conocimientos prácticos, esenciales, que le servirían para un mejor desarrollo del cuerpo del alumno, su cuidado e higiene personal y el de la comunidad (SEP, 1935, p. 210).

En el curso los ejes base, anatomía, fisiología e higiene, los alumnos veían los temas integrados, ubicándolos desde la vida diaria, su tendencia era eminentemente preventiva ("higiénica") y de aplicación. Dándoles a los alumnos, para el caso del conocimiento de la anatomía, lo necesario para que comprendieran la fisiología y para que, partiendo de hechos científicos, dieran sentido a las reglas o preceptos higiénicos, que habrían de aprender para formar hábitos (ver tabla 3). El ideal de la nueva enseñanza estaba impregnada del prgamatismo de Dewey y de la escuela práctica de Makarenko, por ello se pedía que:

[...] para la educación socialista el conocimiento científico no se imparte únicamente como simple información cultural...desea formar generaciones activas y justicieras que sepan siempre utilizar la ciencia y la cultura en beneficio colectivo...por esto les da unidad y las agrupa en los conocimientos sobre la naturaleza, centro de la actividad de la humanidad. (SEP, 1935, p. 205)

Sin embargo dada la formación de los maestros, la mayoría profesionistas libres habilitados, la enseñanza era verbalista, con poco apoyo en los laboratorios -por falta de ellos- y con el dibujo como tecnología didáctica:

[...] un expresivo Ancona nos llena de dibujos a colores el pizarrón y nos transporta en el carro de la imaginación a los siglos anteriores en que se discutían por los naturalistas de esa época los graves problemas de la generación espontánea; un serio y talentoso Rulfo se esfuerza por meternos en nuestra dura cabeza los principios básicos de la Biología y las áridas fórmulas químicas de los compuestos orgánicos; un sereno y paciente Ayala nos conduce de la mano, armada con bisturí o tijeras, para realizar las primeras disecciones de animales vertebrados, en tanto que en la clase de Raíces Griegas, Latinas y Autóctonas Aplicadas a las Ciencias Biológicas, repite una y otra vez Graeci quattuor et virginti litteras habent, o bien nos dedica a analizar la conocida fábula griega de Asinus leonis pelle indutus per campos currebat (Herrejón, 1961). 
Bio - grafía. Escritos sobre la Biología y su Enseñanza. ISSN 2027-1034

Edición Extraordinaria. p.p. 909 - 917

Memorias del IX Encuentro Nacional de Experiencias en Enseñanza de la Biología y la Educación Ambiental. IV Congreso Nacional de Investigación en Enseñanza de la Biología.

Esta enseñanza que se daba a maestros ellos lo replicaban en las escuelas secundarias, la enseñanza de las ciencias biológicas fortalecía lo filosófico y conceptual, pero priorizando como beneficiar al alumno que en su gran mayoría no habrá de seguir cursos superiores (SEP, 1935, p. 230). Con lo anterior, la escuela socialista buscaba el conocimiento concreto de los seres animales y vegetales del país para su aprovechamiento y desarrollar una mentalidad abierta, libre de prejuicios para explicar e interpretar el mundo, pero la falta de condiciones para contar con laboratorios y conocimiento práctico del medio, hacía que la principal forma de enseñanza fuera el discurso oral.

\section{Tabla 3:}

Contenidos sintéticos del curso de Ciencias biológicas III Anatomía fisiología e higiene (SEP, 1935).

\begin{tabular}{lll}
\hline \multicolumn{2}{c}{ Ciencias biológicas III Anatomía fisiología e higiene 3 hrs } \\
\hline $\begin{array}{l}\text { I Introducción } \\
\begin{array}{l}\text { II Conformación general } \\
\text { del cuerpo }\end{array}\end{array}$ & V La digestión & IX Calor animal \\
$\begin{array}{l}\text { III Movilidad del cuerpo } \\
\text { humano }\end{array}$ & VII La respiración & XI Higiene Sexual \\
IV La actividad nerviosa & $\begin{array}{l}\text { VIII La formación de } \\
\text { secreciones y excreciones } \\
\text { urinarias }\end{array}$ & $\begin{array}{l}\text { XII Las grandes campañas } \\
\text { tóxicos enfermedades y }\end{array}$ \\
\hline
\end{tabular}

\section{ANÁLISIS}

Sobre la filosofía. La escuela socialista se asoció a un pensamiento materialista radical. Los aspectos ontológicos, epistemológicos y teológicos fueron abordados cuidadosamente en el diseño del plan de estudios para apoyar la ideología socialista. En lo ontológico, estudiaban las cosas de mundo natural, sus características (anatomía y fisiología), procesos (evolución) y relaciones (ecología) de los seres vivos, incluido el hombre, esto daba la base del enfoque biologicista propuesto por Beltrán. En lo epistemológico, se daba prioridad a la práctica y a lo útil, la forma de aprender, de conocer los seres vivos era a partir de su utilidad (pragmatismo), apoyado en el empirismo, se podía conocer aquello que se podía observar. En lo teológico, lo que habla 


\section{Bio - grafía. Escritos sobre la Biología y su Enseñanza. ISSN 2027-1034}

Edición Extraordinaria. p.p. 909 - 917

Memorias del IX Encuentro Nacional de Experiencias en Enseñanza de la Biología y la

Educación Ambiental. IV Congreso Nacional de Investigación en Enseñanza de la Biología.

sobre el por qué existe lo que es, en este caso los seres vivos. Se tenía un enfoque materialista, se daban explicaciones del origen de la vida y de su devenir sin invocar fuerzas fantásticas. La materia era suficiente para explicar la evolución de la materia. Se develan así los aspectos de estudio de lo sensible para los sentidos, de hablar de procesos de cambio desde la evolución.

Sobre la pedagogía. Definida la orientación, empirista, materialista y utilitaria, la forma de aprender que se postulaba era consistente con estas, ya que se basaba en la experiencia y en la práctica, por ello la forma de enseñar promovía el aprender haciendo de la escuela de la acción, (pragmática) era el sustento para la enseñanza contextuada en busca de un aprendizaje útil más que significativo. Por ello se recomendaba que se vieran los temas de Ciencias biológicas con un enfoque evolucionista, pero priorizando la intención de conocer para explotar, para primar el criterio fundamental, el económico. Para descentrar el individualismo propio del pragmatismo, se fomentaba el trabajo en equipos para la práctica de una acción colectiva prosocial.

Sobre la organización de los contenidos Ledesma (1998) señala que la Biología como ciencia autónoma tiene cuatro paradigmas fundamentales: la teoría celular, teoría de la evolución, la genética y el principio de la homeostasis. En el programa no se atiende teoría celular como tal, se ejemplifica con organismos unicelulares en los dos cursos de biología en sí (botánica y zoología), en el curso de Anatomía fisiología e higiene no se aborda, sino como tejidos. Lo fundamental de la teoría celular, es que toda célula procede de otra célula y esta es la unidad anatómica fisiológica de la vida, el primer sentido no se ve en los contenidos, pero si el segundo. La teoría de la evolución, no se atiende como tema específico en el programa de Ciencias Biológicas, sino como enfoque, se señala de forma reiterada en sus principios y en las orientaciones para el trabajo escolar que los temas deberán de verse con un enfoque evolutivo.

La genética, en los años treinta era una ciencia en ciernes, su relación con la teoría darwinista estaba en construcción. La ciencia de punta estaba lejos de ser atendida en cursos de educación básica.

La homeostasis, entendida como un conjunto de procesos que previenen fluctuaciones en la fisiología de un organismo, fue conceptualizada por Bernard, ahora su idea de auto regulación se ha extendido a la regulación de variaciones en los diversos ecosistemas 0 del universo como un todo (el embrión de la teoría de sistemas). La homeostasis es atendida con un enfoque fisiologista y descuidado en lo biológico, la ecología será una preocupación del diseñador del currículo Enrique Beltrán, pero que no aparece todavía en este programa.

\section{CONCLUSIONES}

El plan de escuela socialista de Cárdenas favorece a la Biología como disciplina que aporte en el pensamiento racional, el enfoque laico y explicaciones materialistas sobre la realidad, pero priorizando conocer la naturaleza para explotarla en beneficio de la sociedad. Durante el cardenismo se hace énfasis en el enfoque evolucionista, una 
Bio - grafía. Escritos sobre la Biología y su Enseñanza. ISSN 2027-1034

Edición Extraordinaria. p.p. 909 - 917

Memorias del IX Encuentro Nacional de Experiencias en Enseñanza de la Biología y la Educación Ambiental. IV Congreso Nacional de Investigación en Enseñanza de la Biología.

perspectiva tan clara y explícita sobre la teoría de la evolución en la educación secundaria en México, no se volvió a dar en este nivel sino hasta 1993. Tomando como referente los paradigmas que señala Ledesma (1999), tres de ellos son atendidos, dos como contenido, homeostasis y teoría celular, uno como enfoque, la teoría de la evolución darwinista y se omitía por ser entonces una teoría emergente la genética. La estructura curricular dual; biológico - naturalista y fisiológico - médico se conserva desde entonces en las subsecuentes propuestas para la enseñanza de la Biología en secundaria hasta nuestros días.

Con todo lo anterior, la escuela socialista buscaba el conocimiento concreto de los seres animales y vegetales del país para su aprovechamiento y el desarrollo de una mentalidad abierta, libre de prejuicios para explicar e interpretar el mundo, desterrar fanatismos, combatir prejuicios, errores y supersticiones y crear hábitos de trabajo e higiene en los alumnos para su beneficio pero sobre todo el de su comunidad. Desafortunadamente la propuesta de enseñanza de las ciencias biológicas fue aprobada hasta 1939 y en México es moda que al cambiar de sexenio se cambie de proyecto educativo, por ello el proyecto cardenista de enseñanza de la ciencia no tuvo sino dos ciclos (dos años) de vida, en 1941 se revierte, pues México podrá tener una educación laica pero su religiosidad era (y es) predominantemente católica, un programa educativo con visos socialistas y materialistas no podría tener una vida larga.

\section{BIBLIOGRAFÍA}

Del Castillo, A. (2000). La polémica en torno a la educación sexual en la Ciudad de México durante la década de los años treinta: conceptos y representaciones de la infancia. Estudios sociológicos. XVIII, 52, 203 - 226.

Gilly, A. (1994). El cardenismo, una utopía. México: Era.

Herrejón, M. (1961) Dos aniversarios paralelos. Sociedad Mexicana de Historia Natural. Disponible en http://repositorio.fciencias.unam.mx:8080/xmlui/bitstream/handle/11154/142752/22 VAniversariosParalelos.pdf?sequence $=1$

IIJ (2009). Constitución política de los Estados Unidos Mexicanos, transitorios de reformas, 1934. México: Instituto de investigaciones jurídicas, UNAM.

INEGI (2004). Estadística. Secretaría de Gobernación. Recuperado el 22 de abril de 2007 de www. inegi.gob. $m x$

Kay - Vaughan, M. (1982). Estado, clases sociales y educación en México. México: SEP/FCE.

Kay - Vaughan, M. (2001). La política cultural en la Revolución. Maestros, campesinos y escuelas en México, 1930-1940. México: FCE. 
Bio - grafía. Escritos sobre la Biología y su Enseñanza. ISSN 2027-1034

Edición Extraordinaria. p.p. 909 - 917

Memorias del IX Encuentro Nacional de Experiencias en Enseñanza de la Biología y la Educación Ambiental. IV Congreso Nacional de Investigación en Enseñanza de la Biología.

Leco T. (2000). La educación Socialista en la Meseta P'urhepecha 1928 - 1940. México: IMCED, Morelia, Michoacán.

Ledesma, I. (1999). El conflicto entre Alfonso L. Herrera e Isaac Ochoterena y la institucionalización de la biología en México. Tesis de doctorado en ciencias. Facultad de ciencias de la UNAM.

Maldonado, A. (2008). Una educación para el cambio social 1928 - 1940. México: Universidad Michoacana de San Nicolás de Hidalgo. Morelia, Michoacán

Montes de Oca, E. (2007). La educación en México: Los libros oficiales de lectura editados durante el gobierno de Lázaro Cárdenas, 1934-1940. Perfiles educativos, 29 (117), 111-130.

Reyes, C. (1983). La educación indígena durante el cardenismo. México: CREFAL

SEP (1934). Memorias relativas al estado que guarda el ramo de educación pública el 31 de agosto de 1934, Tomo I, exposición. Documentos SEP. México: SEP.

SEP (1935). Memoria relativa al estado que guarda el ramo de educación pública. El 31 de agosto de 1935. Tomo II. Documentos SEP. México: SEP.

SEP (1941). La educación pública en México, desde el $1^{\circ}$ de diciembre de 1934 hasta el 30 de noviembre de 1940. Tomo II. Documentos SEP. México: SEP.

Ynclán, G. (Coord.). (1998). Todo por hacer. México: Patronato para la cultura del maestro, A.C.

Zorrilla, M. (2004). La educación secundaria en México, al filo de su reforma. REICE, Revista electrónica iberoamericana sobre calidad, eficacia y cambio en educación. Vol. $\quad 2 \quad N^{\circ} \quad 1 . \quad 1$ Disponible $\quad$ en http://www.ice.deusto.es/RINACE/reice/vol2n1/Zorrilla.pdf 